\title{
Estilo de vida de universitários: Análise de estudantes em diferentes turnos
}

\author{
University students' lifestyle of students in diferent shifts
}

Estilo de vida en estudiantes universitarios: Análisis de estudiantes em diferentes horario de estudio

Recebido: 25/04/2021 | Revisado: 01/05/2021 | Aceito: 03/05/2021 | Publicado: 16/05/2021

\author{
Amanda Lopes dos Santos \\ ORCID: https://orcid.org/0000-0003-1172-0786 \\ Universidade Estadual do Paraná, Brasil \\ E-mail: amaaandalopez16@gmail.com \\ Sérgio Roberto Adriano Prati \\ ORCID: https://orcid.org/0000-0003-4375-2467 \\ Universidade Estadual do Paraná, Brasil \\ E-mail: srap@bol.com.br \\ Aline de Souza Santos \\ ORCID: https://orcid.org/0000-0001-8265-6828 \\ Universidade Estadual do Paraná, Brasil \\ E-mail: alinedesouza2690@gmail.com.br
}

\begin{abstract}
Resumo
Analisar o estilo de vida de universitários de diferentes turnos. Foram investigados 400 estudantes de uma universidade de Paranavaí-PR, 287 de Administração e 113 de Pedagogia; todos responderam ao questionário Estilo de vida Fantástico (EVF), composto por 25 questões, subdividido em 9 dimensões: Família e amigos (F); Atividade física (AF); Nutrição (N); Tabaco e drogas (TD); Álcool (Al); Segurança, estresse e atividade sexual (S); Tipo de comportamento (TC); Introspecção (I) e Carreira (C). Cada resposta atribui um valor de 0 a 4 pontos, totalizando escore máximo de 100 (quanto mais alto, melhor o estilo de vida). Em média os estudantes de Administração obtiveram $65,0 \pm 10,4$ pts e estudantes de pedagogia $67,6 \pm 10,9$ pts. Na comparação entre estudantes de turnos diferentes observou-se que não houve diferença estatística $(\mathrm{P}>0,05)$ entre estudantes de pedagogia (Vespertino, $\mathrm{n}=56, \mathrm{EVF}=$ $67,8 \pm 10,7$ pts e Noturno, n=57, EVF= 67,4 $\pm 11,2$ pts), em contrapartida estudantes de administração apresentaram diferença $(\mathrm{P}=0,01)$, sendo que estudantes do turno matutino $(68,1 \pm 9,3)$ apontaram melhor EVF em relação ao noturno $(64,1 \pm 10,6 \mathrm{pts})$. Por fim, foi identificado que $35 \%$ dos estudantes de Administração alcançaram o escore mínimo (70pontos) considerado mais saudável e de menor risco, sendo $28 \%$ do período matutino e $71 \%$ do noturno. Quanto aos estudantes de Pedagogia, $45 \%$ obtiveram EVF considerado adequado, sendo $43 \%$ do vespertino e $56 \%$ do noturno. O Estilo de vida dos universitários é predominantemente inadequado; estudantes do período noturno apresentam pior estilo de vida quando comparado com estudantes diurnos e isso é mais evidente em estudantes do sexo masculino.
\end{abstract}

Palavras-chave: Estudantes; Estilo de vida; Comportamento de risco; Saúde.

\begin{abstract}
To analyze the university students' lifestyle of different shifts. It was instigated 400 students of a university in Paranavaí, 287 of Business and 113 of Pedagogy. All of them answered the questionnaire Fantastic Lifestyle, composed by 25 questions, subdivided into 9 dimensions: Family and friends (F); Physical activity (PA); Nutrition (N); Tobacco and drugs (TD); Alcohol (Al); Safety, stress and sexual activity (S); Type of behavior (TC); Introspection (I) and Career (C). Each answer assigns a value from 0 to 4 points, totaling a maximum score of 100 (the higher the score, the better the lifestyle). On average, the Business students obtained $65.0 \pm 10.4$ pts, Pedagogy students reached $67.6 \pm 10.9$ pts. In the comparison between students of different shifts it was observed that there was no statistical difference ( $\mathrm{P}>0.05$ ) among students of Pedagogy (Afternoon, $\mathrm{n}=56, \mathrm{EVF}=67.8 \pm 10.7$ pts and Night $\mathrm{n}=57$, EVF 67,4 \pm 11 ,2pts), whereas Business students showed some difference $(\mathrm{P}=0,01)$, the morning shift $(68.1 \pm 9.3)$ presented better results than the night one $(64.1 \pm 10.6 \mathrm{pts})$. Finally, it was identified that $35 \%$ of Business students reached the minimum score (70 points) considered healthier and of lower risk, with $28 \%$ morning students and $71 \%$ night. As for Pedagogy students, $45 \%$ obtained EVF considered adequate, being $43 \%$ of afternoon and $56 \%$ of night. College students' lifestyle is predominantly inadequate; night students' lifestyle is worse than day students and this is most evident in male students.
\end{abstract}

Keywords: Students; Lifestyle; Risk-taking; Health.

\section{Resumen}

Analizar el estilo de vida de estudiantes universitarios de diferentes turnos. Se investigaron 400 estudiantes de una universidad de Paranavaí-PR, 287 de Administración y 113 de Pedagogía; todos respondieron el cuestionario Fantastic Lifestyle (25 preguntas) subdivididas en 9 dimensiones: Familia y amigos (F); Actividad física (AF); 
Nutrición (N); Tabaco y drogas (TD); Alcohol (Al); Seguridad, estrés y actividad sexual (S); Tipo de comportamiento (TC); Introspección (I); Carrera (C). Cada respuesta asigna un valor de 0 a 4 puntos, totalizando una puntuación máxima de 100 (cuanto más alto, mejor es el estilo de vida). En promedio, los estudiantes de Administración obtuvieron $65.0 \pm 10.4$ pts y los estudiantes de pedagogía $67.6 \pm 10.9$ pts. Al comparar estudiantes de diferentes turnos, se observó que no hubo diferencia estadística $(\mathrm{P}>0.05)$ entre los estudiantes de Pedagogía (Tarde, $\mathrm{n}=56, \mathrm{EVF}=67.8$ $\pm 10.7 \mathrm{pts}$; Nocturne, $\mathrm{n}=57, \mathrm{EVF}=67.4 \pm 11.2 \mathrm{pts}$ ), por otro lado, los estudiantes de Administración mostraron una diferencia $(\mathrm{P}=0.01)$, y los estudiantes del turno de la mañana $(68.1 \pm 9.3)$ mostraron un mejor EVF en relación al turno de la noche $(64,1 \pm 10.6 p t s)$. Finalmente, se identificó que el 35\% de los estudiantes de Administración alcanzó la puntuación mínima (70 puntos) considerada más saludable y menos riesgosa, 28\% estudiando en la mañana y $71 \%$ en la noche. En cuanto a los estudiantes de Pedagogía, el $45 \%$ obtuvo EVF considerada adecuada, el $43 \%$ de los cuales por la tarde y el $56 \%$ por la noche. El estilo de vida de los estudiantes universitarios es predominantemente inadecuado; los estudiantes nocturnos tienen un estilo de vida peor en comparación con los estudiantes diurnos, esto es más evidente en los estudiantes varones.

Palabras clave: Estudiantes; Estilo de vida; Asunción de Riesgo; Salud.

\section{Introdução}

O estilo de vida para Añez, Reis e Petroski (2008) é caracterizado por padrões de comportamento e práticas cotidianas que implicam efeito considerável na saúde da população e está associado a inúmeros aspectos que refletem os valores, as ações e as oportunidades na vida das pessoas ${ }^{1}$. Estas práticas segundo Nahas (2006) estão ligadas à compreensão de qualidade de vida do indivíduo, podendo ser mudadas com o passar dos anos, e para isso acontecer é preciso conscientemente perceber algum valor em comportamentos que devem ser incluídos ou não, além de identificar que é capaz de realizar as mudanças pretendidas.

Ainda sobre o estilo de vida, ele pode ser entendido também como um conjunto de comportamentos que evidenciam um dos principais articuladores quando se trata dos níveis da qualidade de vida e saúde das pessoas. Entre estes comportamentos, existem aqueles que podem prejudicar negativamente os níveis de saúde e são considerados comportamentos de risco à saúde (CRS), como o uso do tabaco, consumo exacerbado de bebidas com álcool, hábitos alimentares nãoadequados, consumo de drogas ilícitas, atos sexuais sem prevenção e níveis insuficientes de atividade física entre outros. Esses temas têm sido constantemente investigados em diferentes populações, dentre elas os estudantes universitários (Farias Júnior \& Lopes, 2008).

Em um estudo de Carvalho, Fonseca, Barbosa, Machado, Santos e Silva. (2015) foi constatado que os universitários representavam um público cujo estilo de vida e situações próprias do meio acadêmico podiam resultar em diversos CRS, como a falta de refeições, alto consumo de lanches rápidos e inadequados quanto ao valor nutricional. Além disso tudo, alta taxa de sedentarismo, excesso de peso e fatores de risco cardiovascular nesse grupo populacional. No Brasil, segundo os autores, existe uma grande prevalência destes fatores de risco em estudantes do ensino superior, como excesso de peso $(38,1 \%)$, de sedentarismo (34,8\%), de hipercolesterolemia (27,5\%), de tabagismo $(14,6 \%)$ e de HAS (hipertensão arterial sistêmica) em universitários $(8,4 \%)$.

De acordo com Souza e Borges (2016), o período acadêmico na universidade é caracterizado por alterações relevantes no desenvolvimento psíquico e social, há um aumento das necessidades alimentares e a obrigação do indivíduo em satisfazer essas necessidades. É durante essa fase que os hábitos ruins em relação à alimentação e também o sedentarismo estão muito presentes, resultando em grande prejuízo à saúde e desempenho acadêmico desses universitários.

Quando se trata de estilo de vida negativo de estudantes do ensino superior, não se pode descartar o uso do álcool, que apresenta dados em estudo em relação ao seu consumo (Silva; Pereira, Almeida, Silva \& Oliveira, 2012). Quando se refere à ingestão de álcool pelos universitários, a preocupação aumenta levando em consideração que o álcool é admitido, e de certa forma incentivado pela sociedade. 
Em uma investigação realizada com estudantes universitários mostrou-se que, para esse grupo populacional, ao ingerir bebida alcoólica estariam fugindo de problemas cotidianos. Seria uma saída para lidar com situações negativas resultantes de aspectos sociais e econômicos, além de ser uma alternativa para lidar com situações negativas de caráter pessoal principalmente pelo prazer que a bebida traz ou a situação social em que ela é consumida. Quanto aos dados sobre a quantidade e frequência de doses de bebida alcoólica ingeridas, observou-se o quão alarmante são os números encontrados, visto que mais da metade dos entrevistados (53\%) tinha o hábito de beber toda semana (Barros, Barros, Bernardes, Lima \& Silva, 2012).

Outro fator de grande importância no estilo de vida dos universitários é o sono, mesmo que algumas pesquisas exponham o relevante papel do sono no processo de aprendizagem, observou-se, uma tendência a má qualidade de sono entre os estudantes de graduação. Os autores apontam o estresse, a tensão, a depressão, o consumo exagerado de energéticos e bebidas alcoólicas e o uso excessivo das redes sociais como possíveis razões para essa condição. Isso talvez se deva pelo excesso de atividade na qual são expostos os universitários, em especial àqueles que atuam em três turnos, ou seja, estudam no período noturno, pois trabalham ao longo do dia (Obrecht et al., 2015). A importância do sono ganha destaque e notabilidade quando se constatam os efeitos negativos causados pela privação do mesmo, como, por exemplo, déficit cognitivo, alteração de humor, fadiga e identificação na execução de tarefas do dia a dia (Souza, Santos, Moraes, Silva \& Ellinger, 2018).

No Brasil é comum as instituições de ensino superior ofertarem cursos em diferentes turnos para os alunos. Pesquisa mostra que universitários do período noturno apresentam maiores queixas de sono, menos horas dormidas e cansaço. Enquanto $39,4 \%$ obtiveram índice de ter um sono bom, mais da metade dos alunos $(60,6 \%)$ obtiveram a classificação de sono ruim. O motivo que explica esse resultado seria que os estudantes que frequentam o turno da noite, em sua maioria, trabalham durante o dia, o que aumentaria a demanda de responsabilidades e diminuiria o tempo para cumpri-las comprometendo seu estilo de vida e saúde geral (Obrecht et al, 2015).

Acredita-se que o fato do turno ser determinante para problemas com o sono de universitários, pode ser que isso também interfira na questão do estresse, da ansiedade, da fadiga impedindo a prática de atividades e exercícios físicos, ou até mesmo na rotina com atividades de lazer e convívio social fora do trabalho e estudo. Diante dessas questões, o presente trabalho objetiva analisar o estilo de vida de estudantes universitários de diferentes turnos.

\section{Metodologia}

O presente estudo tem caráter descritivo e exploratório na qual coleta e analisa informações sem intervir, sendo essas informações ainda pouco conhecidas (Thomas, Nelson \& Silverman, 2012). A pesquisa foi aprovada pelo Comitê de Ética da Universidade Estadual de Maringá sobre registro 61069916.8.0000.0104 e parecer nº. 2.093.464 de 2017.

A população foi composta por todos os estudantes de Administração e Pedagogia regularmente matriculados em uma instituição de ensino superior pública do município de Paranavaí-PR, tendo a amostra formada por 400 universitários sendo 287 do curso de Administração e 113 do curso de Pedagogia, alunos que foram favoráveis em participar da pesquisa voluntariamente, bem como responderam ao questionário proposto no momento da coleta de dados.

Para determinação do estilo de vida foi utilizado o questionário Estilo de Vida (EV) Fantástico (Añez et al, 2008), um instrumento composto por 25 questões, sendo 2 dicotômicas (respostas com valor 0 ou 4) e 23 com respostas que variam de 0 a 4 pontos, sendo subdividido em 9 domínios de EV ( $\mathrm{F}=$ família; $\mathrm{AF}=$ atividade física; N=nutrição; $\mathrm{TD}=$ tabaco e drogas; $\mathrm{AL}=$ ingestão de álcool; $\mathrm{S}=$ =comportamento preventivo e estresse; TC=padrão de comportamento geral; I=introspecção; $\mathrm{C}=$ carreira e satisfação profissional;).

A coleta foi realizada em sala de aula com a presença de todos os alunos que aceitarem participar da pesquisa. O instrumento questionário de EV Fantástico é auto-explicativo, porém os pesquisadores estavam presentes em sala para 
orientação inicial para o preenchimento do formulário. Cada aluno identificou em seu formulário nome, série e turno do curso que frequentava, bem como, sexo e data de nascimento. Depois de respondidas as questões os escores eram somados para se identificar a classificação de EV dos estudantes. Os resultados podem variar de 0 a 100 pontos, sendo quanto maior o valor melhor EV. Para análise, além da pontuação geral, os resultados foram estratificados isoladamente por domínio. Nesse sentido foram considerados os resultados de forma dicotomizada sendo valores 0,1 e 2 considerando como "inadequados" (EV não saudável/maior risco) e respostas 3 e 4 como "adequados" (EV saudável/menor risco).

Para análise de dados, foi utilizada a estatística descritiva com valores expressos em percentual, média e desvio padrão geral, por domínio do estilo de vida, por sexo e por série. Foi usado para verificar diferenças entre escores no EV dos estudantes de diferentes turnos, por sexo e por série os testes paramétricos "t" de Student análise de variância (ANOVA). Em todos os testes estatísticos o grau de confiança foi de $95 \%$.

\section{Resultados e Discussão}

O objetivo do trabalho foi analisar o estilo de vida em universitários de diferentes turnos. Para melhor organização, os resultados foram apresentados em forma de tabela, sendo que a Tabela 1 apresenta resultados do estilo de vida dos universitários separados por turno com valores em média, desvio padrão e porcentagem dos alunos que apresentaram estilo de vida considerado seguro e saudável ( $\geq 70 \mathrm{pts}$ ). A seguir, na Tabela 2 são expostos os resultados do estilo de vida dos universitários separados por domínios, com valores em média, desvio padrão e porcentagem dos alunos que apresentaram estilo de vida considerado seguro e saudável ( $\geq 70$ pts). Por fim, na Tabela 3 são expostos os resultados do estilo de vida dos universitários separados por domínios, com valores em média e desvio padrão dos alunos separados por turnos (matutino, vespertino, diurno e noturno).

Tabela 1 - Estilo de vida de universitários. Análise entre estudantes de diferentes turnos e cursos. Teste " $\mathrm{t}$ " e ANOVA, valores em média, desvio padrão e percentual de casos adequados. Nível de confiança de 95\%:

\begin{tabular}{|c|c|c|c|}
\hline $\begin{array}{l}\text { Turnos } \\
\text { no }\end{array}$ & $\overline{E V F}$ & $\% \mathrm{EVF}$ & $\mathbf{P}$ \\
\hline \multicolumn{4}{|l|}{ TURNOS... } \\
\hline$\overline{\text { Noturno }(n}=185)$ & $65,0 \pm 11.3$ & 39 & $*$ \\
\hline Diurno $(n=115)$ & $67,9 \pm 10.0$ & 44 & $*$ \\
\hline Matutino $(\mathrm{n}=59)$ & $68,1 \pm 9,3$ & 49 & \\
\hline Vespertino $(\mathrm{n}=56)$ & $67,8 \pm 10,7$ & 39 & \\
\hline \multicolumn{4}{|c|}{ CURSOS } \\
\hline Administração & $65,0 \pm 1,0$ & 35 & * \\
\hline Matutino & $68,1 \pm 9,3$ & 28 & \\
\hline Noturno & $64,1 \pm 1,0$ & 71 & \\
\hline Pedagogia & $67,6 \pm 1,0$ & 45 & \\
\hline Vespertino & $67,8 \pm 10,7$ & 43 & \\
\hline Noturno & $67,4 \pm 1,1$ & 56 & \\
\hline \multicolumn{4}{|l|}{ SEXOS.. } \\
\hline Masculino (n=119) & $63,1 \pm 1,2$ & 20 & \\
\hline Feminino $(n=281)$ & $67,1 \pm 1,0$ & 35 & \\
\hline \multicolumn{4}{|l|}{ Diurno.... } \\
\hline Masculino (n=18) & $65,6 \pm 8,5 \#$ & 38 & \\
\hline Feminino $(n=95)$ & $68,5 \pm 9,9 \#$ & 45 & \\
\hline \multicolumn{4}{|l|}{ Noturno..................... } \\
\hline Masculino (n=51) & $62,2 \pm 1,3 \#$ & 33 & $*$ \\
\hline Feminino $(n=134)$ & $66,0 \pm 1,0 \#$ & 41 & $*$ \\
\hline
\end{tabular}

*Teste “t”, significativo quando $\mathrm{P}<0,05$; \#ANOVA, significativo quando $\mathrm{P}<0,05$. Fonte: Autores. 
A tabela acima apresenta os resultados quanto ao estilo de vida dos universitários, separados por turno do curso, com valores em média, desvio padrão e porcentagem dos alunos que apresentaram estilo de vida considerado seguro e saudável ( $\geq 70$ pts). Nota-se diferença entre valores de estudantes de diferentes turnos.

$\mathrm{Na}$ comparação entre estudantes de turnos diferentes, estudantes do diurno $(67,9 \pm 10,03)$ apresentaram melhor EVF $(\mathrm{P}<0,05)$ em relação ao noturno $(65,0 \pm 11,3)$. É provável que essa diferença entre o EVF dos universitários seja decorrente

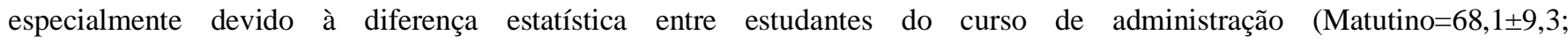
Noturno=64,1 $\pm 1,0, \mathrm{P}=0,01)$, visto que não foi observada diferença estatística em estudantes de pedagogia $(\mathrm{P}>0,05)$. Estes resultados corroboram com o estudo de Loch e Nahas (2006) que analisou estudantes do ensino médio, no qual os autores observaram as diferenças nas proporções entre o turno que o estudante frequentava e os comportamentos negativos relacionados à saúde, constatando que os adolescentes do período noturno apresentavam consideravelmente maior de consumo inadequado de frutas e se declaravam fumantes, sendo que nesta variável a proporção observada foi mais que o dobro no turno noturno em comparação com o diurno.

Outro aspecto que confirma as diferenças entre turnos é decorrente da comparação entre cursos, na qual estudantes de Administração, predominantemente universitários noturnos (228 alunos) tiveram uma pontuação menor que atingiu em média $65,0( \pm 1,0)$ pts em relação aos universitários de Pedagogia, que obtiveram uma média de 67,6 $( \pm 1,0)$ pts $(\mathrm{P}=0,02)$. Embora a pontuação do curso de Pedagogia tenha sido em média maior $(\mathrm{P}<0,05)$ que a do curso de Administração, vale ratificar que ambos os cursos ficaram abaixo da média que se adotou como um EV adequado (EVF $\geq 70$ pts). No estudo de Silva et al (2012), realizado com calouros de uma universidade, constatou-se que 5,1\% dos calouros apresentaram estilo de vida inadequado, isso mostra que, as pessoas de um modo geral, possuem hábitos de vida diferentes do que se considera ideal, embora haja evidências em relação às atitudes que promovem um estilo de vida saudável e os efeitos dele decorrentes.

$\mathrm{Na}$ análise de variância (ANOVA), quando se comparou os três grupos (matutino, vespertino e noturno), não houve diferença entre turnos $(\mathrm{P}>0,05)$. Entretanto, quando comparados os turnos diurno masculino $(65,6 \pm 8,5 \mathrm{pts})$ e feminino (68,5 $\pm 9,9$ pts) e noturno masculino $(62,2 \pm 1,3$ pts) e feminino $(66,0 \pm 1,0$ pts), houve diferença $(\mathrm{P}<0,05)$ sendo o período da noite classificado como um pior EV, bem como o sexo masculino também justificando esses resultados. Em uma investigação feita por Araújo e Almondes (2012) em estudantes de diferentes turnos, foi constatado que os níveis de sonolência dos universitários que estudavam no período da noite, eram significativamente $(\mathrm{P}<0,05)$ maiores em relação aos estudantes da manhã, considerando que $68 \%$ dos alunos do turno da noite trabalhavam. Nos estudos de Lourenço, Souza, Fonseca, Virtuoso Júnior e Barbosa (2016) procurando investigar comportamento sedentário em estudantes universitários, foi encontrado que, com maior suscetibilidade, os alunos do período de estudo noturno apresentam menos envolvimento com a atividade física (48,8\%), no entanto, obtiveram menor probabilidade de estarem expostos ao uso de computador e tempo de tela. Acredita-se que este resultado esteja intimamente relacionado às características do grupo que normalmente apresentam as obrigações de trabalho e que estas podem não estar relacionadas ao uso do computador.

Em relação à comparação por sexo, não houve diferença estatística $(\mathrm{P} \geq 0,05)$ entre homens que estudam no período diurno $65,6( \pm 8,5)$ pts quando comparados com o período noturno $62,2( \pm 1,3)$ pts. Isso acontece na comparação entre mulheres também. Já quando comparados ambos os sexos, os homens (62,2 $\pm 1,3$ pts) que estudam do período noturno têm um pior estilo de vida em relação ao das mulheres $(66,0 \pm 1,0)$ do mesmo turno $(\mathrm{P}<0,05)$. Ao pesquisar o nível de atividade física da população de São Paulo, o estudo de Matsudo et al (2002) apontou que porcentagem de mulheres (48,6\%) ativas fisicamente é maior que a dos homens (42,5\%) justificando que pelo menos nesse aspecto o estilo de vida delas seria melhor que do masculino. Farias Júnior et al (2009), ao analisar comportamentos de risco (CRS) em adolescentes do sul do Brasil constatouse que adolescentes do sexo masculino apresentaram maior prevalência de relação ao baixo consumo de frutas e verduras, demonstraram consumo abusivo de álcool, consumo de drogas ilícitas e o envolvimento em brigas. Dentre os mais jovens, as 
moças, por sua vez, compreenderam maior prevalência de níveis insuficientes de atividade física e quanto ao comportamento sexual de risco (a não utilização de preservativos regularmente nas relações sexuais).

Diante desses resultados é importante identificar em quais aspectos os universitários estariam adotando um pior comportamento e estilo de vida, contribuintes para desenvolvimento de doenças e riscos aumentados à saúde geral, visto que no ambiente acadêmico torna-se comum o envolvimento de jovens com comportamentos de risco e suscetibilidade maior a eles, assim, na Tabela 2 as análises do EVF compreenderão as dimensões do questionário em questão.

Tabela 2 - Estilo de vida de universitários. Análise entre estudantes de diferentes turnos e cursos por dimensão do estilo de vida. Comparação entre turnos: diurno x noturno (teste " $t$ "); e entre matutino x vespertino x noturno (ANOVA). Valores em média, desvio padrão e percentual de casos adequados (quando respostas 3 ou 4 para cada questão do EVF). Confiança de 95\%:

\begin{tabular}{|c|c|c|c|c|c|c|c|}
\hline $\begin{array}{l}\text { Turnos - } \\
\text { Dimensões }\end{array}$ & $\begin{array}{l}\text { TODOS } \\
\text { unos } n=40\end{array}$ & $\overline{E V F}$ & $\begin{array}{c}\text { MAT\%EVF } \\
\text { n=59 }\end{array}$ & $\begin{array}{c}\text { VES\%EVF } \\
n=56\end{array}$ & $\begin{array}{c}\text { DIU\%EVF } \\
n=115\end{array}$ & $\begin{array}{c}\text { NOT\%EVF } \\
n=185\end{array}$ & $\overline{\mathbf{P}}$ \\
\hline$\overline{F(\geq 6)}$ & $6,5 \pm 1,7$ & 79 & 84 & 85 & 84 & 76 & \\
\hline$A(\geq 6)$ & $2,6 \pm 2,3$ & 37 & 15 & 13 & 14 & 11 & \\
\hline$N(\geq 9)$ & $5,8 \pm 2,6$ & 15 & 18 & 20 & 19 & 13 & \\
\hline$T(\geq 13)$ & $14,1 \pm 1,8$ & 85 & 87 & 85 & 86 & 84 & \\
\hline$A(\geq 10)$ & $10,4 \pm 2,4$ & 80 & 76 & 92 & 84 & 77 & * \\
\hline$S(\geq 15)$ & $13,4 \pm 3,5$ & 41 & 47 & 40 & 43 & 39 & * \\
\hline$T(\geq 6)$ & $3,9 \pm 1.8$ & 17 & 11 & 17 & 14 & 19 & \\
\hline I $(\geq 9)$ & $7,2 \pm 2,4$ & 32 & 35 & 37 & 36 & 29 & \\
\hline$C(\geq 3)$ & $2,7 \pm 1,1$ & 64 & 74 & 69 & 71 & 59 & * \\
\hline
\end{tabular}

MAT - Matutino; VES - Vespertino; NOT - Noturno. F=família; AF=atividade física; N=nutrição; TD=tabaco e drogas; AL=ingestão de álcool; $\mathrm{S}=$ comportamento preventivo e estresse; $\mathrm{TC}=$ padrão de comportamento geral; $\mathrm{I}=$ introspecção; $\mathrm{C}=$ carreira e satisfação profissional. (*Teste "t" e \#ANOVA quando $\mathrm{P}<0,05)$. Fonte: Autores.

A Tabela 2 apresenta os resultados do estilo de vida dividido pelos domínios que compõem o instrumento, os valores são apresentados em média, desvio padrão e porcentagem dos alunos que apresentaram estilo de vida considerado seguro e saudável ( $\geq 70$ pts). Diferença entre os domínios é encontrada ao analisar os estudantes de administração e pedagogia de todos os turnos, apenas em 3 dimensões os pesquisados apresentaram valor indicando estilo de vida adequado, valor acima de 70pts.

De uma forma geral, observa-se que em média, apenas nas dimensões família e amigos (F), uso de tabaco e drogas (T) e uso de álcool (A) o EVF dos estudantes atingiu os critérios mínimos de segurança. A preocupação aumenta ao se observar que nas dimensões atividade física $(\mathrm{A})$, nutrição $(\mathrm{N})$ e tipo de comportamento $(\mathrm{T})$ as médias ficaram muito abaixo do critério considerado ideal e mais saudável do EVF, bem como o percentual de casos adequados, por dimensão foi sempre os menores respectivamente 37\%, 15\% e 17\%. Esse resultado se assemelha com estudo de Rovida, Sumida, Santos, Moimaz e Garbin (2015) que verificou o estresse e estilo e vida em acadêmicos ingressantes, onde os resultados apontaram que $12 \%$ dos alunos (baixa porcentagem) incluem frutas e verduras regularmente em suas refeições, 32\% tem o hábito de evitar alimentos gordurosos e $64 \%$ fazem menos de quatro refeições variadas por dia, incluindo o café da manhã completo. Mendes, Silva, Messia, Carvalho e Silva (2016) retratou que estudantes universitários não seguem geralmente hábitos alimentares saudáveis, a alimentação típica deste público é rica em gordura e insuficiente em frutas e vegetais. Estes resultados condizem com a pesquisa feita pela vigilância de fatores de risco e proteção para doenças crônicas por inquérito telefônico (2015), na qual verificou o baixo consumo destes alimentos saudáveis pela população brasileira, apontando apenas $20,2 \%$ de consumo adequado deste grupo de alimentos (Brasil, 2015). 
Tabela 3 - Estilo de vida de universitários. Comparação entre turnos: diurno x noturno (teste " $t$ "); e entre matutino x vespertino x noturno (ANOVA). Valores em média e desvio padrão. Confiança de 95\%:

\begin{tabular}{|c|c|c|c|c|c|}
\hline $\begin{array}{l}\text { Turnos } \\
\text { Dimensões }\end{array}$ & $\begin{array}{l}\text { MAT } \\
n=59\end{array}$ & $\begin{array}{l}\text { VES } \\
n=56\end{array}$ & $\begin{array}{l}\text { DIU } \\
n=115\end{array}$ & $\begin{array}{l}\text { NOT } \\
n=185\end{array}$ & $\mathbf{P}$ \\
\hline$\overline{F(\geq 6)}$ & $6,7( \pm 1.6)$ & $6,7( \pm 1,5)$ & $6,7( \pm 1,5)$ & $6,4( \pm 1,7)$ & \\
\hline$A(\geq 6)$ & $2,9( \pm 2,3)$ & $2,6( \pm 2,4)$ & $2,7( \pm 2,3)$ & $2,5( \pm 2,5)$ & \\
\hline$N(\geq 9)$ & $6,3( \pm 2,4)$ & $5,9( \pm 2,6)$ & $6,1( \pm 2,5)$ & $5,6( \pm 2,6)$ & \\
\hline$T(\geq 13)$ & $1,3( \pm 1,4)$ & $1,4( \pm 1,8)$ & $1,4( \pm 1,6)$ & $1,4( \pm 1,9)$ & \\
\hline$A(\geq 10)$ & $1,0( \pm 2,1)$ & $1,1( \pm 2,0)$ & $1,0( \pm 2,1)$ & $1,0( \pm 2,5)$ & $*$ \\
\hline$S(\geq 15)$ & $1,4( \pm 3,7)$ & $1,3( \pm 3,3)$ & $1,3( \pm 3,5)$ & $1,3( \pm 3,7)$ & $*$ \\
\hline$T(\geq 6)$ & $4,0( \pm 1,7)$ & $4,1( \pm 1,7)$ & $4,0( \pm 1,7)$ & $3,9( \pm 1,9)$ & \\
\hline I $(\geq 9)$ & $7,8( \pm 2,0)$ & $7,3( \pm 2,8)$ & $7,6( \pm 2,4)$ & $7,0( \pm 2,4)$ & \\
\hline$C(\geq 3)$ & $2,8( \pm 1,0)$ & $3,0( \pm 0,9)$ & $2,9( \pm 1,0)$ & $2,6( \pm 1,1)$ & $*$ \\
\hline
\end{tabular}

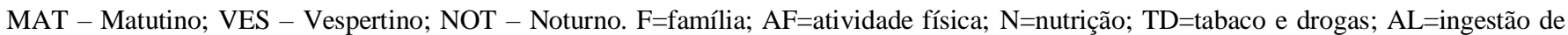
álcool; $\mathrm{S}=$ comportamento preventivo e estresse; $\mathrm{TC}=$ padrão de comportamento geral; $\mathrm{I}=$ introspecção; $\mathrm{C}=$ carreira e satisfação profissional. (*Teste "t" e \#ANOVA quando $\mathrm{P}<0,05)$. Fonte: Autores.

Por último, na Tabela 3 são expostos os resultados do estilo de vida dos universitários (valores em média e desvio padrão) separados por domínios e por turnos (matutino, vespertino, diurno e noturno). É possível perceber que foram atingidos valores menos em relação ao período noturno, quando comparado aos outros períodos.

De acordo com os dados apresentados, o domínio Atividade Física obteve um resultado indesejável na qual $37 \%$ dos avaliados atingiram a média de 70pts. Esse resultado fica mais preocupante quando são separados por turnos, sendo o período noturno o que atingiu a menor porcentagem em estudantes com o estilo de vida adequado (11\%). Pode-se observar que a maioria dos acadêmicos declarou exercer trabalho remunerado. Esses resultados se comprovam, em comparação à estudo que investigou fatores associados ao nível de atividade física entre estudantes universitários e constatou a questão socioeconômica interfere na questão da inatividade física, pois, o fato dos acadêmicos investigados frequentarem cursos no período noturno tende a facilitar e garantir a oportunidade de ter um trabalho remunerado, ainda assim, a rotina de trabalhar durante o dia e estudar durante a noite pode dificultar a prática de atividade física devido à falta de tempo livre (Vargas, Cartorani, Vargas e Gutierrez, 2015).

No domínio uso de Álcool, $80 \%$ dos universitários atingiram o escore mínimo adotado como um estilo de vida adequado e com menor risco ( $\geq 70$ pts). Porém, na comparação entre turnos (diurno e noturno) houve diferença estatística $(\mathrm{P}<0,05)$, sendo que os universitários que estudam no período diurno $6,7( \pm 1.0)$ são considerados melhores que os estudantes do período noturno 6,5 $( \pm 1,1)(\mathrm{P}=0.04)$. Numa pesquisa de Lemos et al (2007) sobre uso de substâncias psicoativas, fez-se um levantamento entre universitários da Bahia avaliando o consumo de álcool e outras drogas revelou que dentre os estudantes de medicina, 87,10\% já haviam feito uso de álcool. Em pesquisa mais recente, Evangelista, Kadooka, Pires e Constantino (2018) ao analisar padrões e consumo de álcool em estudantes universitários, destacou que 70\% dos estudantes já ficaram embriagados com o consumo de alguma bebida alcoólica e entre os tipos de bebidas mais consumidas, a cerveja ou chope tiveram o percentual mais alto, 64,4\% e por sequência as bebidas destiladas (uísque, gim, vodca, rum, conhaque, pinga/cachaça/aguardente, tequila ou batidas) com 59,5\%.

Ao analisar o domínio Comportamento Preventivo e Estresse, observou-se que houve uma pequena diferença $(\mathrm{P}=0.05)$ entre os universitários que estudam no período diurno quando comparados aos do período noturno, na qual os estudantes da noite atingiram uma média de 13,1 ( $\pm 3,5)$ pts ficando abaixo dos alunos que estudam durante o dia 13,9 ( $\pm 3,5)$. No geral, os universitários estão abaixo do escore esperado para um estilo de vida saudável e seguro, $41 \%$ dos analisados atingiram pontos $\leq 70$. Em outro estudo, ao analisar o estilo de vida de acadêmicos de Educação Física, obteve um resultado parecido sendo que $66 \%$ estavam inadequados ${ }^{6}$. Em Passo Fundo, Rio Grande do Sul, observou que dos universitários 
investigados 55,7\% não usavam camisinha nas relações sexuais. Desta forma, é válido ressaltar a importância de instituir medidas de conscientização para posterior mudança de atitudes, objetivando evitar doenças sexualmente transmissíveis e gestação indesejada (Opperman, Gassen, Fracasso e Rosa (2002).

Quando comparado o domínio Carreira e Satisfação Profissional, observou-se que houve diferença estatística $(\mathrm{P}=0,02)$ entre os períodos diurno e noturno. $71 \%$ dos investigados do período diurno $2,9( \pm 1.0)$ pts. obtiveram um EV adequado, enquanto 59\% dos investigados do período noturno 2,6 ( $\pm 1,1)$ pts. Estes resultados estão em concordância com um estudo sobre uso de substâncias psicoativas em ingressantes de enfermagem, no qual a maioria dos estudantes $(76,7 \%)$ sente-se acelerado e/ou atarefado algumas vezes, e em torno da metade desses estudantes (50,8\%) admitiam às vezes estar aborrecido e/ou agressivo. Em contrapartida, a maioria desses acadêmicos $(58,9 \%)$ sente-se quase sempre feliz com o que faz e as atividades em geral (Virgínio, 2015). Em contrapartida, outra pesquisa constatou que 54,8\% dos acadêmicos de Educação Física mostraram-se insatisfeito com o trabalho ou a função que exerciam. A suposição é de que esses resultados estejam associados ao fato de que a maior parte dos estudantes entrevistados não trabalhava de modo remunerado e talvez por isso desejarem um emprego formal (Silva et al, 2012).

\section{Conclusão}

Estilo de vida dos universitários é predominantemente inadequado, e estudantes do período noturno apresentam estilo de vida em pior condição quando comparados com estudantes diurnos. Isso é mais evidente em estudantes do sexo masculino.

Os estudantes apresentaram pior EV nos domínios Ingestão de álcool, comportamento preventivo e estresse, e por fim, carreira e satisfação profissional.

A mudança que ocorre quando o adolescente passa a ser um jovem adulto é uma etapa da vida muito importante para a definição de hábitos comportamentais saudáveis ou não, e, estes comportamentos poderão permanecer ao longo da vida. Portanto, é de suma importância agir de forma ativa na educação das pessoas e das comunidades, principalmente junto dos estudantes do ensino superior, oferecendo-lhes recursos que os possibilitem escolhas saudáveis, ou seja, promover estilos de vida saudáveis e potencializando ganhos em segurança e saúde.

A partir dos achados nesse estudo, sugere-se que uma maior população seja investigada, afim de apontar quais as demais influências que possam existir entre universitários em relação aos diferentes horários dos cursos de graduação, e que trabalhos futuros possam investigar quais as possibilidades de amenizar os comportamentos de risco presentes nesses indivíduos.

\section{Referências}

Añez, C. R. R., Reis, R. S. \& Petroski, E. L. (2008). Versão brasileira do questionário "estilo de vida fantástico": tradução e validação para adultos jovens. Arquivos Brasileiros de Cardiologia, 91(2), 102-9.

Araújo, D. F. \& Almondes, K. M. (2012). Avaliação da sonolência em estudantes universitários de turnos distintos. Psico-USF, 17(2), $295-302$.

Barros, C. V. L., Barros, D. A. C., Bernardes, M. J. C., Lima, W. V. \& Silva, L. C. S. (2012). A influência do convívio universitário na adesão ao alcoolismo. Itinerarius Reflectionis, 2(13).

Brasil - Vigitel. (2015). Vigilância de fatores de risco e proteção para doenças crônicas por inquérito telefônico. http://tabnet.datasus.gov.br/cgi/vigitel /vigteldescr.htm

Carvalho, C. A., Fonseca, P. C. A., Barbosa, J. B., Machado, S. P., Santos, A. M. \& Silva, A. A. M. (2015). Associação entre fatores de risco cardiovascular e indicadores antropométricos de obesidade em universitários de São Luís, Maranhão, Brasil. Ciência \& Saúde Coletiva, 20(2), 479-490.

Evangelista, V. M. A., Kadooka, A., Pires, M. L. N. \& Constantino, E. P. (2018). Padrões e consumo de álcool entre estudantes universitários. Revista Psicologia, Diversidade e Saúde, 7(2), 192-204.

Farias Junior, J. C. \& Lopes, A. S. (2008). Comportamentos de risco relacionados à saúde em adolescentes. Revista Brasileira de Ciência e Movimento, 12(1), 7-12. 
Farias Júnior, J. C., Nahas, M. V., Barros, M. V. G., Loch, M. R., Oliveira, E. S. A. \& De Bem, M. F. L. (2009). Comportamentos de risco à saúde em adolescentes no Sul do Brasil: prevalência e fatores associados. Revista Panamericana de Salud Pública, 25(4), 344-352.

Lemos, K. M., Neves, N. M. B. C., Kuwano, A. Y., Tedesqui, G., Bitencourt, A. G. V., Neves, F. B. C. S., Guimarães, A. N. Rebello, A., Bacellar, F. \& Lima, M. M. (2007). Uso de substâncias psicoativas entre estudantes de Medicina de Salvador (BA). Revista de psiquiatria clínica, 34(3), $118-124$.

Look, M. R. \& Nahas, M. V. (2006). Comportamentos negativos relacionados A saúde em estudantes do ensino médio de Florianópolis, SC. Revista Brasileira de Atividade Física \& Saúde, 11(2), 13-24.

Lourenço, C. L. M., Souza, T. F., Fonseca, A. S., Virtuoso Junior, J. S. \& Barbosa, A. R. (2016). Comportamento sedentário em estudantes Universitários. Revista Brasileira de Atividade Física \& Saúde, 21(1), 67-77.

Matsudo SM, Matsudo VR, Araújo T, Andrade D, Andrade E, Oliveira, L. \& Braggion, G. (2002). Nível de atividade física da popu lação do Estado de São Paulo: análise de acordo com o gênero, idade, nível socioeconômico, distribuição geográfica e de conhecimento. Revista Brasileira de Ciência e Movimento, $10(4), 41-50$

Mendes, M. L. M., Silva, F. R., Messia, C. M. B. O., Carvalho, P. G. S. \& Silva, T. F. A. (2016). Hábitos alimentares e atividade física de universitários da área de saúde do município de Petrolina-PE. Tempus Actas de Saúde Coletiva, 10(2), 205-217.

Nahas, M. V. (2006). Atividade física, saúde e qualidade de vida: conceitos e sugestões para um estilo de vida ativo. Midiograf.

Oppermann, K., Gassen, D. T., Fracasso, J. I. \& Rosa, L. C. (2002). Postura dos universitários de Passo Fundo em relação à contracepção e prevenção de DSTs. Revista AMRIGS, 46(3,4), 146-150.

Rovida, T. A. S., Sumida, D. H., Santos, A. S., Moimaz, S. A. S. \& Garbin, C. A. S. (2015). Estresse e o estilo de vida dos acadêmicos ingressantes em um curso de graduação em Odontologia. Revista da ABENO, 15(3), 26-34.

Obrecht, A., Collaço, I. A. L., Valderramas, S. R., Miranda, K. C., Vargas, E. C. \& Szkudlarek, A. C. (2015). Análise da qualidade do sono em estudantes de graduação de diferentes turnos. Revista Neurociências, 23(2), 205-210.

Silva, D. A. S., Pereira, I. M. M., Almeida, M. B., Silva, R. J. S. \& Oliveira, A. C. C. (2012). Estilo de vida de acadêmicos de educação física de uma universidade pública do estado de Sergipe, Brasil. Revista Brasileira de Ciências do Esporte, 34(1), 53-67.

Souza, F. N., Santos, I. J. L., Moraes, S. R., Silva, C. M. S. \& Ellinger, V. C. M. (2018). Associação entre qualidade do sono e excesso de peso entre estudantes de medicina da Universidade Severino Sombra, Vassouras-RJ. Almanaque Multidisciplinar de Pesquisa, 1(2), 100-113.

Sousa, K. J. Q. \& Borges, G. F. (2016). Estilo de vida, atividade física e coeficiente acadêmico de universitários do interior do amazonas-brasil. Revista Brasileira de Ciências da Saúde, 20(4), 277-284.

Thomas, J. R., Nelson, J. K. \& Silverman, S. J. (2012). Métodos de pesquisa em atividade física. Artmed.

Vargas, L. M., Cartorani, J. R. H., Vargas, T. M. \& Gutierrez, G. L. (2015). Fatores associados ao nível de atividade física entre estudantes universitários. Revista Brasileira de Qualidade de Vida, 7(3), 158-168.

Virgínio, A. F. C. (2015). Estilos de Vida - Consumo de substâncias psicoativas dos “caloiros” de Enfermagem. Dissertação (Mestrado) - Escola Superior de Enfermagem de Coimbra - Portugal, 1-148. 